\title{
Un regard nouveau sur la prospérité. L'émergence des statistiques économiques au Canada (1891-1911)
}

\section{Jean-Pierre Beaud et Jean-Guy Prévost}

Numéro 18-19, 1992

URI : https://id.erudit.org/iderudit/1002312ar

DOI : https://doi.org/10.7202/1002312ar

Aller au sommaire du numéro

\section{Éditeur(s)}

Département de sociologie - Université du Québec à Montréal

ISSN

0831-1048 (imprimé)

1923-5771 (numérique)

Découvrir la revue

Citer cet article

Beaud, J.-P. \& Prévost, J.-G. (1992). Un regard nouveau sur la prospérité. L'émergence des statistiques économiques au Canada (1891-1911). Cahiers de recherche sociologique, (18-19), 277-295. https://doi.org/10.7202/1002312ar
Résumé de l'article

Jusqu'à la fin du XIXe siècle, l'activité principale des statisticiens était le recensement des personnes et leur attention se portait essentiellement sur les statistiques de la population. Plus précisément, la croissance démographique était tenue pour l'indicateur privilégié de la prospérité du pays. Le début du XXe siècle correspondra toutefois, pour la plupart des appareils statistiques nationaux, à une transformation profonde : désormais, les données relatives à la production et aux échanges occuperont la première place et les spécialistes de celles-ci, les économistes, prendront les postes de commande. Cet article expose comment cette mutation s'est opérée dans un cas précis, celui du Canada; il s'agira en somme de repérer les transformations que connaîtront certains objets d'enquête, l'apparition de nouveaux champs d'investigation et surtout l'émergence rapide, dans le discours produit par l'appareil statistique, de concepts clés de l'économie politique classique. 
Cahiers de recherche sociologique, nos $18-19,1992$

\section{Un regard nouveau sur la prospérité. L'émergence des statistiques économiques au Canada (1891-1911)}

Jean-Pierre BEAUD et Jean-Guy PRÉVOST*

Dans son rapport accompagnant les résultats du recensement des deux Canadas pour 1851 , le secrétaire du Bureau d'enregistrement et de statistique, William Hutton, cite longuement Sir John Sinclair, auteur d'un monumental Statistical Account of Scotland en vingt et un volumes, suivant lequel les statistiques ont pour objet "de constater le quantum de bonheur dont jouissent" les habitants d'un pays ${ }^{1}$. Cette formule résume admirablement la conception que l'on se faisait au Canada de la collecte de données statistiques depuis le recensement effectué par Jean Talon en 1666 (le tout premier des temps modernes... selon les Canadiens) ${ }^{2}$ jusqu'à la fin du XIXe siècle. On pourrait parler à ce propos de point de vue démographique, puisque l'activité principale des "statisticiens" est alors le recensement des personnes et que leur attention se porte avant tout sur les statistiques de la population. Les appareils statistiques qui se mettent sur pied au cours du siècle dernier sont d'ailleurs, en Grande-Bretagne, en France, en Hongrie, en Norvège, en Italie et à peu près partout ailleurs, dirigés par des démographes ${ }^{3}$. Mais on pourrait également parler de point de vue encyclopédique ou topologique:

* Les auteurs désirent remercier le Conseil de recherches en sciences humaines du Canada et Statistique Canada pour leur aide matérielle; Yohanna Loucheur et Pierre Laprise, respectivement candidats à la maîtrise et au doctorat en science politique à l'UQAM, qui ont participé à la recherche sous-tendant cet article; enfin, les deux évaluateurs anonymes qui ont examiné une première version de ce texte.

1 Ministère de l'Agriculture, Recensement des Canadas 1851-52, vol. I (1853), xl. Notons que Sinclair s'enorgueillissait d'avoir introduit le mot "statistics" dans la langue anglaise.

2 "It is usual to start off the history of Canadian statistics with a bang. The honour of taking the first census of modern times belongs to Canada!" (R. Hamilton Coats, "Beginnings in Canadian Statistics", The Canadian Historical Review , 18 (1946), p. 109).

3 Le Canada constitue toutefois une exception à cette quasi-règle: cette situation s'explique sans doute par la faible autonomie de l'appareil statistique naissant vis-à-vis du pouvoir politique; les nominations y ont un caractère nettement partisan. Sur cette période, voir l'ouvrage de J. et M. Dupâquier, Histoire de la démographie, la statistique de la population des origines à 1900, Paris, Perrin, 1985. 
le recensement canadien de 1871 , par exemple, le premier à suivre la naissance de la Confédération en 1867, est animé par un idéal d'exhaustivité, une passion de tout savoir sur tout; il ne diffère guère de ces almanachs produits par des "gentlemen-statisticiens" comme Joseph Bouchette ou Robert Gourlay, inventaires imposants dans lesquels se combinent, sous une forme pas nécessairement numérique, renseignements géographiques, historiques et démographiques et où l'on peut entendre un écho très net de la vieille statistik allemande ${ }^{4}$. Le début du $\mathrm{XXe}$ siècle correspondra toutefois, pour la plupart des organisations statistiques nationales, à une transformation profonde: désormais, ce sont les données relatives à la production et aux échanges qui occuperont la première place et les spécialistes de celles-ci, les économistes, qui prendront peu à peu les postes de commande dans les institutions statistiques. Cette prise en charge par le groupe des économistes sera certes plus complète et plus apparente quelques décennies plus tard, lorsque plusieurs pays se lanceront dans la mise sur pied d'une comptabilité nationale et lorsque, sous l'influence des idées keynésiennes, la nécessité d'une gestion macroéconomique sera admise, appelant la mise au point d'un nombre impressionnant de séries sur le chômage, les revenus, le coût de la vie et plusieurs autres variables économiques. Mais bien avant cela, le Statisticien du Dominion Robert Hamilton Coats pouvait déjà écrire que "le gouvernement (...) est une agence unique dont le devoir suprême est de guider la politique économique" et que "les méthodes peu sûres du passé étant caduques, les statistiques (devaient) de plus en plus devenir le guide du gouvernement ${ }^{5}$. . Dès le tournant du siècle en effet, les statistiques relatives à la réalité économique prendront une importance croissante, relativisant la place jusqu'alors quasi exclusive qu'occupait le recensement des personnes.

Nous voudrions exposer ici comment cette mutation qui a affecté l'ensemble des appareils statistiques nationaux à la même époque s'est opérée dans le cas précis du Canada; il s'agira en somme de repérer les transformations que connaîtront certains objets d'enquête, l'apparition de nouveaux champs d'investigation, mais surtout l'émergence rapide, dans le discours produit par l'appareil statistique, de concepts clés de l'économie politique classique. L'intérêt du cas canadien tient à ce que l'appareil statistique étant à cette époque peu développé, il est passablement aisé d'observer les formes particulières qu'y a prises cette mutation. Avant 1891,

4 Bouchette, qui occupait les fonctions d'arpenteur général du Bas-Canada, a publié en 1815 une Description topographique de la province du Bas-Canada avec des remarques sur le Haut-Canada et sur les relations des deux provinces avec les États-Unis de l'Amérique. En 1831, il fit paraître une étude en trois volumes intitulée The British Dominions in North America. Gourlay publia pour sa part en 1822 un Statistical Account of Upper Canada Compiled with a View to a Grand System of Emigration in Connexion with a Reform of the Poor Laws. L'ouvrage comprend trois forts volumes, si l'on inclut l'imposante introduction de 500 pages publiée séparément.

5 R. Hamilton Coats, A National System of Statistics for Canada (1916), p. 6; Dominion Bureau of Statistics, First Annual Report of the Dominion Statistician for the Fiscal Year Ended March 31, 1919. The Dominion Bureau of Statistics Its Origin, Purpose and Organization, 1919, p. 7. 
on ne peut pas vraiment parler de statistiques économiques pour la simple raison qu'il n'existe alors aucun lien entre cette discipline théorique qu'est la science économique et cette activité pratique qu'est la collecte de données relatives à des phénomènes empiriques auxquelles la première s'intéresse: plus précisément, on n'observe pas, avant cette date, ce travail d'abstraction et de conceptualisation qui seul témoignerait de l'émergence d'un point de vue nouveau. Certes, on dresse des tableaux ayant trait aux occupations, aux établissements industriels ou à la valeur des instruments agricoles. Mais cette cueillette de renseignements s'inscrit tout à fait dans la logique encyclopédique qui prévaut alors: il s'agit de procéder à un décompte de la richesse du pays, de mesurer sa prospérité; le choix des indicateurs retenus pour ce décompte ne découle en aucune manière d'une réflexion théorique. On assiste en revanche au cours des décennies 1890 et 1900 au Canada à une véritable mise en forme statistique des concepts de l'économie politique classique qui commencent tout juste à circuler dans l'univers intellectuel canadien: cette importation d'un bagage théorique nouveau dans le discours statistique obligera à une critique du travail antérieur, notamment en matière de définitions et de découpages, et rendra possible un déplacement de perspective ainsi qu'une focalisation du travail d'enquête autour de l'idée de production. Non pas que l'on sente tout à coup le besoin de prendre la mesure de la prospérité du pays (cela, on le fait depuis toujours), mais, plutôt, la discipline alors naissante offre un langage permettant d'envisager la mesure de cette prospérité comme une analyse du développement de la production. Certes, les années 1890-1914 ont vu un développement considérable de l'industrie au Canada: il y a effectivement des réalités nouvelles ou du moins plus aisément perceptibles; les commentaires des responsables du recensement témoignent, comme on le verra, d'une conscience nette de ces changements. En ce sens, il y a conjonction entre les transformations socio-économiques que connaît le pays (notamment la multiplication et la concentration des industries et les changements entraînés par celles-ci dans la distribution de la main-d'œuvre) et la disponibilité d'un langage conceptuel et technique nouveau, à l'aide duquel on cherchera à les saisir. Jusqu'à la fin du siècle dernier, le principal indicateur de la richesse était, en quelque sorte par défaut, la population totale: c'est le rythme d'accroissement de cette dernière qui était constamment invoqué comme mesure ultime de la prospérité, plutôt que des variables, à nos yeux beaucoup plus "économiques", comme l'occupation ou l'industrie, dont la définition et le découpage ne font l'objet d'aucune réflexion systématique avant 1890 . Ce que permettra entre autres l'introduction des concepts de l'économie politique dans le discours statistique, c'est précisément de tracer une démarcation plus nette entre ce que l'on appellera désormais les statistiques économiques et les statistiques vitales.

La période cruciale à cet égard est celle qui va grosso modo du recensement de 1891 à celui de 1911. Notons que pour l'un des rares historiens du système statistique canadien, l'économiste M.C. Urquhart, la première de ces décennies n'existe pour ainsi dire pas. Il écrit en effet qu'après le départ de Joseph-Charles 
Taché "there is no evidence of any other substantial figure in the development of Canadian statistics (...) until Archibald Blue arrived in Ottawa in 19006". En fait, le nom de George Johnson, Chief Government Statistician à partir de 1887, n'apparaît nulle part dans l'article d'Urquhart. Celui-ci, qui s'intéresse aux "bâtisseurs" du système statistique canadien et envisage la question sous l'angle institutionnel, est certes autorisé à négliger ainsi Johnson, qui, contrairement à Taché, Blue et Coats, n'a pas réussi à mener à bien la réforme qu'il jugeait nécessaire. Cette omission demeure toutefois surprenante lorsqu'on sait à quel point Coats, statisticien du Dominion de 1915 à 1942 et à ce jour principal historien de la collecte de données statistiques au Canada, s'est lui-même attaché à mettre en valeur la contribution de Johnson au développement des statistiques canadiennes, insistant sur la vision centralisatrice dont celui-ci se serait fait l'avocat et que son successeur Blue n'aurait pas adoptée avec suffisamment d'énergie ${ }^{7}$. Bien sûr, Coats n'est pas un témoin impartial: il n'est pas exclu qu'il ait cherché à diminuer l'importance de son prédécesseur immédiat pour donner plus de relief à sa propre contribution tout en situant celle-ci dans une tradition dont il ne se présentait que comme le continuateur fidèle et consciencieux ${ }^{8}$. Mais ces réserves admises, il nous semble, quoi qu'en pensent Urquhart et Coats, qu'un examen plus poussé de "la période Johnson" et de "la période Blue" s'impose, non pas tant pour des raisons d'ordre institutionnel (il est exact que le projet de réforme de Johnson n'a existé que sur papier) que pour des raisons d'ordre conceptuel: c'est en effet au cours de ces deux décennies que les préoccupations, les objets et les concepts propres à la science économique pénétreront le discours statistique, donnant à celuici un visage qu'il conserve encore aujourd'hui.

\section{Statistique, bonheur et prospérité}

Le but des statistiques, écrivait donc Sinclair, est de mesurer "le quantum de bonheur" dont jouissent les habitants du pays. L'interrogation qui traverse tout le rapport de Hutton est en effet la suivante: la masse des données recueillies (et il convient de rappeler que, malgré ses défauts indéniables, ce recensement de 1851 fut le premier à couvrir les deux Canadas en même temps et au moyen des mêmes questionnaires, que son rapport d'une centaine de pages et la mise en forme de tableaux s'étendant sur près de mille pages tranchaient nettement sur les bilans congrus auxquels donnaient lieu les recensements précédents), cette masse de renseignements témoigne-t-elle de la prospérité croissante du Canada? Ou plus précisément, puisque c'est en termes comparatifs que le problème est toujours

6 M. C. Urquhart, "Three Builders of Canada's Statistical System", Canadian Historical Review, 68 (1987), p. 419.

7 Voir notamment R.H. Coats, "Fifty Years of Statistical Progress", in Fifty Years Retrospect: Canada 1882-1932, Toronto, 1932, p. 78-79.

8 Sur ce point, voir J.-P. Beaud et J.-G. Prévost, "La structuration de l'appareil statistique canadien (1912-1921)", communication présentée au congrès annuel de l'Association canadienne de science politique, mai 1992. 
posé, permet-elle de réfuter "cette idée (qui) existe très généralement non seulement dans la métropole mais même en Canada, que le développement et la prospérité de ce pays ne sont pas comparables à ceux des États-Unis (... $)^{9 "}$ ? Tout le sens de l'investigation à laquelle vient de se livrer le Bureau est justement d'apporter une réponse à cette question.

Quels sont les renseignements recueillis qui permettraient d'indiquer "les progrès d'une colonie où les éléments du développement agissent avec une énergie si croissante et presque sans exemple ${ }^{10 "}$ ? Notons d'abord que Hutton est conscient des insuffisances du recensement à cet égard: ainsi, il regrette que l'on ait négligé de s'enquérir de la valeur des instruments agricoles, "critérium important de la richesse industrielle $^{11 " ~(c e t t e ~ l a c u n e ~ s e r a ~ c o r r i g e ́ e ~ l o r s ~ d u ~ r e c e n s e m e n t ~ s u i v a n t, ~ e n ~ 1861) ; ~ i l ~}$ note également, faisant des "objets de luxe" un indicateur important de la prospérité du pays, que l'on aurait dû relever le nombre de voitures d'agrément ${ }^{12}$; enfin, il se désole de la mauvaise qualité du recensement des moulins et manufactures qui ne donne "qu'une information très limitée sur la richesse de la colonie", puisqu'on n'y a pas distingué loyer, capital et profit net, ce qui rend difficile l'étude de leur valeur $^{13}$. Mais le critère auquel s'en remet Hutton lorsque vient le temps de prouver que "le développement du Haut-Canada, à partir de l'année 1800, (a) été trois fois plus grand que celui des États-Unis ${ }^{14 ",}$ c'est la croissance de la population. Pour démontrer son assertion, Hutton invoque notamment les calculs suivants: si, de 1800 à 1851, la population des États-Unis a augmenté de 400\%, celle du Canada, pour une période plus courte, soit de 1811 à 1851 , a crû de $1100 \%$; même en prenant pour points de comparaison ceux des États américains qui se peuplent le plus rapidement (Ohio, Michigan, Illinois), le Haut-Canada sort gagnant avec une croissance de $375 \%$ pour la période $1830-1849$, contre $320 \%$ pour les États susmentionnés de 1830 à 1850; si l'on se tourne maintenant vers les grandes villes, on constate qu'au cours des années 1840-1850, Boston a vu sa population s'accroître de $45 \%$ alors que Toronto a connu une progression de $95 \%$ qui lui permet de devancer également New York, Saint-Louis et Cincinnati; pour la dernière décennie, le Canada bat à plate couture les États-Unis et la Grande-Bretagne (Hutton n'hésite d'ailleurs pas à donner dans le chauvinisme lorsqu'il s'agit de vanter les performances de la colonie: en 10 ans, "le Haut-Canada a fait le même progrès que l'Angleterre en onze siècles"). Non seulement "les statistiques du Canada prouvent qu'il règne ici le même esprit que dans les États-Unis", mais, pour ce qui est de l'Ohio, cet État étalon choisi par Hutton parce qu'il constitue le cas le plus favorable aux États-Unis, "ne pouvons-nous pas raisonnablement espérer que nous le laisserons bien loin dans l'ombre au prochain recensement

9 Ministère de l'Agriculture, Recensement des Canadas 1851-52, vol. I, p. x-xi.

10 Ibid., p. iv.

11 Ibid., p. viii.

12 "On reconnaîtra que cet item est très important en réfléchissant que ce sont des objets de luxe provenant du superflu de moyens des propriétaires (...)" (ibid., p. ix).

13 Ibid., p. ix-x.

14 Ibid., p. xi. Souligné par l'auteur. 
décennal ${ }^{15}$ "? La croissance démographique est donc la preuve du développement et de la prospérité de la colonie.

Un tel plaidoyer, un tel télescopage entre politique et statistique font aujourd'hui sourire; mais on voit bien, à la lecture, quel type d'argument pouvait à l'époque emporter la conviction ou, pour dire les choses autrement, ce qui comptait le plus dans ce que l'on comptait. C'est d'ailleurs de cette façon, comme le révèle la lecture des débats de la Chambre des communes, que les députés liront le décompte de la population, jusqu'à la fin du siècle à tout le moins. Le passage du principe de dénombrement de facto au principe de jure à partir de 1871 devait certes introduire une nouvelle dimension, explicitement politique celle-là, dans l'appréciation des résultats des recensements: l'article 51 de l'Acte de l'Amérique du Nord britannique prévoyait en effet que la représentation parlementaire de chaque province devait être révisée et fixée à la suite de chaque recensement décennal, ce qui obligeait le Canada à renoncer à la méthode de facto, en vigueur dans la métropole britannique, au profit de la méthode de jure, utilisée par les voisins américains, chez qui la représentation des États au Congrès était également déterminée par les résultats du recensement ${ }^{16}$. Tous les recensements réalisés de 1871 à 1901 feront l'objet de critiques sévères à la Chambre des communes, quel que soit le parti au pouvoir. Ainsi, le leader libéral Sir Richard Cartwright utilisera à propos du recensement de 1881 un cliché peu flatteur: "il ne vaut pas le papier sur lequel il est écrit ${ }^{17 "}$. Le reproche le plus fréquent porte justement sur la méthode de jure dont on prétend qu'elle conduit à une surévaluation de la population en certains districts, puisqu'elle

15 Ibid., p. xii, et xxiii.

16 Selon les Dupâquier, la notion de population de droit (de jure), qui recouvrait les personnes ayant domicile légal même si elles étaient temporairement absentes de leur résidence habituelle, a dominé la pratique des recensements dans la première moitié du XIXe siècle. Devant les problèmes que posait l'inclusion ou non de la population flottante, c'est la Belgique qui la première, suivant en cela les recommandations des congrès internationaux de statistiques, adopta la notion de population de fait (de facto), qui recouvrait les personnes présentes le jour du recensement (Histoire de la démographie, p. 325). Pour les pays dont la représentation territoriale était constitutionnellement liée aux résultats du recensement, il était toutefois impossible de se soustraire au mode de jure, quels qu'en fussent les inconvénients. Sur l'expérience américaine, voir M. Anderson, The American Census, a Social History, Madison, Wisconsin University Press, 1988. Il n'est pas clair par ailleurs que les recensements de 1851 et 1861 aient été véritablement menés suivant la méthode de facto. Le ler mars 1882, le ministre de l'Agriculture Pope répondant à une question du député Blake déclare: "Je dois dire (...) que des recensements du Canada, depuis 1841 jusqu'à 1861, ont été faits en inscrivant tout le monde que l'on trouvait. On inscrivait tous les étrangers de même que les absents" (Débats des Communes, 1882, p. 176).

17 Ibid., p. 1162. Vingt ans plus tard, les libéraux seront au pouvoir, Cartwright sera ministre du Commerce et l'opposition conservatrice ne manquera pas de dire que "les masses de ce pays ne considèrent pas les chiffres produits dans de telles circonstances comme ayant la moindre valeur". (Débats de la Chambre des communes, 1902, p. 4125). 
incite les énumérateurs à inscrire des personnes qui ont quitté le pays sans espoir de retour $^{18}$. Ces critiques atteindront un sommet lorsque le journaliste conservateur George Johnson sera nommé Chief Government Statistician en $1887^{19}$. Pour l'opposition libérale, ce "monsieur qui a été employé à la littérature électorale du gouvernement" et "qui consacre une bonne partie de son temps à préparer des documents pour servir aux discours des députés de la droite" ne mérite "pas beaucoup de créance ${ }^{20 "}$. Dans les débats qui précèdent le recensement de 1891, des voix se font à nouveau entendre qui reprochent au mode de jure d'être "un mode imaginaire qui attribue au Canada une population imaginaire", puisque "l'énumérateur est le juge pour décider si le nom d'une personne doit être inscrit ou non, et les énumérateurs diffèrent beaucoup d'opinion sur ce point". On ajoute généralement que ces énumérateurs étant payés au nom, il y va de leur intérêt d'en inscrire le plus possible ${ }^{21}$. La réponse des députés du parti gouvernemental à ce genre de critique tourne pour sa part autour du fait que le passage au mode de facto pénaliserait indûment les provinces de l'Atlantique et le Québec dont un bon nombre de citoyens travaillent hors de leur province et seraient de ce fait absents le jour du recensement ${ }^{22}$. Mais ce n'est pas ce problème de représentation parlementaire qui suscite les critiques de l'opposition; c'est plutôt que le mode de jure permet au gouvernement de camoufler tout à la fois l'important mouvement de Canadiens vers les États-Unis et l'échec de sa politique économique, jugée responsable de cette émigration massive ${ }^{23}$. En effet, "le gouvernement prétend que

18 Voir par exemple les remarques du député Blake en 1882: "Avec ce système de recensement, personne ne peut dire quel est le chiffre véritable de notre population" (Débats des communes, 1882, p. 176); ou celles du député Davies en 1890: "(...) avec le mode que le gouvernement se propose d'adopter, nous aurons un dénombrement propre à induire considérablement en erreur" (Débats de la Chambre des communes, 1890, p. 2440). Il est à noter qu'une fois au pouvoir les libéraux, pourtant ardents avocats de la méthode de facto lorsqu'ils étaient dans l'opposition, invoqueront l'argument de la continuité pour fins de comparaison et se convertiront à la méthode de jure. (Débats de la Chambre des communes, 1900 , p. 8481).

19 G. Johnson (1837-1911) fut editor du Halifax Reporter, puis correspondant à Ottawa du Toronto Mail. Commissaire du recensement de 1881 pour la Nouvelle-Écosse, il sera nommé Chief Statistician au ministère de l'Agriculture en 1887 et portera le titre de Dominion Statistician à partir de 1891. Il sera une des bêtes noires de l'opposition libérale qui verra sa nomination comme une récompense politique et lui reprochera de faire le travail d'un propagandiste du gouvernement conservateur. Lorsque les libéraux prendront le pouvoir en 1896, Johnson restera en poste, mais la responsabilité du recensement de 1901 lui sera retirée pour être confiée à Archibald Blue, qui deviendra de facto le principal statisticien du pays.

20 Débats des Communes, 1888, p. 1702 et 1703.

21 Débats de la Chambre des communes, 1890, p. 2440 et 2439. Le député Casey: "Il est également possible que le prix payé à l'énumérateur pour chaque nom a pu être pour quelque chose dans le nombre de noms qu'il a inscrits" (p. 2443).

22 Ibid., p. 2445-2446. 
sa politique a eu l'effet de garder les Canadiens dans le pays et de leur donner de l'emploi dans le pays même", mais en adoptant le mode de jure, il refuse de fournir les renseignements qui permettraient de juger une telle affirmation, puisque de cette façon, "on a inscrit les noms de plusieurs centaines et plusieurs milliers de personnes qui avaient quitté le Canada, s'étaient établies dans un autre pays et s'étaient fait naturaliser aux États-Unis ${ }^{24 "}$. La croissance démographique ne nous renseigne donc pas seulement sur un phénomène de population: elle constitue également l'indicateur privilégié du succès ou de l'échec de la politique économique du gouvernement.

À cet égard, le document le plus explicite est sans doute le discours prononcé par Cartwright le 6 août 1891. S'appuyant sur le premier résultat consécutif au recensement d'avril 1891 et qui fixe la population globale du Canada à 4289344 âmes, le leader de l'opposition se lance dans une impressionnante philippique contre le gouvernement conservateur. Alors que, quarante ans plus tôt, William Hutton croyait pouvoir s'appuyer sur la croissance démographique du Canada pour affirmer que celui-ci "laissait loin dans l'ombre" la mère patrie et même "son entreprenant voisin", Cartwright déplore le retard du Canada sur les États-Unis et le fait que "nous nous trouvions réduits au niveau des vieux pays, sous le rapport de l'augmentation de la population". Confrontant le total résultant du dénombrement effectué en 1891 à celui de 1881 et aux estimations annuelles produites par le ministère de l'Agriculture (présentées par lui comme de pures falsifications destinées à masquer la stagnation du pays), Cartwright y voit non seulement un taux d'accroissement très inférieur à celui des États-Unis, mais aussi la preuve que la plupart des immigrants venus au Canada pendant la dernière décennie l'ont quitté au profit des États-Unis, de même que de nombreux Canadiens appartenant à "notre population la meilleure et de premier choix". Selon Cartwright, les données disponibles démontrent que "durant ces douze dernières années, il n'y a eu (...) aucune augmentation réelle de la richesse de la population ${ }^{25 "}$. Tout cela lui permet de dresser un acte d'accusation global contre l'administration conservatrice et de s'en prendre plus particulièrement à sa politique de tarifs douaniers qu'il juge première responsable de ce qu'il présente comme un exode massif. Il est important de noter

23 Sur ce problème de population, on consultera l'ouvrage de Y. Lavoie, L'émigration des Canadiens aux États-Unis avant 1930, Montréal, Presses de l'Université de Montréal, 1972.

24 Débats de la Chambre des communes, 1890, p. 2448 et 2440.

25 Débats de la Chambre des communes, 1891, p. 4937, 4947 et 4942 . La réponse du gouvernement à cette charge sera faite par le ministre des Finances George Foster. Selon lui, les résultats des recensements de 1881 et 1891 sont incomparables, parce qu'on a apporté des modifications au mode d'énumération: alors qu'en 1881 on avait appliqué très libéralement le mode de jure et de ce fait inscrit de nombreuses personnes dont les chances de revenir étaient quasi nulles, tous ceux qui avaient quitté depuis plus d'un an ont été omis en 1891. Pour ne pas être en reste vis-à-vis Cartwright, Foster ajoute que l'exode est dû au dénigrement systématique auquel se livrent les libéraux et plus particulièrement le chef de l'opposition (ibid., p. 4966). 
ici que la charge du leader de l'opposition à l'endroit de la politique économique du gouvernement s'appuie essentiellement sur le décompte de la population: ni sur la main-d'œuvre ni sur la production ne dispose-t-il de renseignements pouvant donner de plus larges dimensions à son réquisitoire.

\section{De la division du travail}

Bien qu'à la Chambre des communes on mesure encore, en 1891, la prospérité du pays à travers le seul prisme de la croissance démographique, c'est de ce recensement que l'on peut précisément dater l'émergence du point de vue économique. Dans le manuel d'instructions destiné aux énumérateurs, le tout nouveau statisticien en chef George Johnson écrit à propos des métiers et professions:

Il y a cinquante ans, le cordonnier faisait la chaussure complète, le tailleur confectionnait tout l'habillement; aujourd'hui, par suite de la subdivision du travail, il est peu d'ouvriers qui commencent et terminent une pièce d'ouvrage. (...) Dans tous les travaux de l'industrie, le spécialiste a remplacé l'ouvrier d'autrefois, et la statistique des professions doit fournir les détails les plus particuliers si l'on veut qu'elle réponde aux besoins ${ }^{26}$.

Cette référence au concept de division du travail est tout à fait nouvelle, du moins en ce qui concerne les métiers et professions ${ }^{27}$. Sur le plan du classement des occupations, le recensement de 1891 marque donc une nette rupture vis-à-vis du passé $^{28}$. Jusque-là, la nomenclature des occupations cherchait en quelque sorte à refléter spontanément l'univers du travail, les recenseurs se limitant à ordonner le matériau brut recueilli auprès des personnes interrogées. Ainsi, lors des recensements de 1851 et 1861 , on s'était contenté de dresser un inventaire des métiers artisanaux: simplement rangées suivant un ordre alphabétique

26 Ministère de l'Agriculture, Manuel contenant l'Acte du recensement" et les instructions aux officiers employés à faire le troisième recensement du Canada , 1891, p. 14.

27 Dans l'introduction au volume III du Recensement du Canada 1871, on lit toutefois: "La division du travail n'est pas en Canada ce qu'elle est dans les vieilles sociétés de l'Europe et souvent le même établissement voit réunies ensemble plusieurs branches d'industries qu'il est en conséquence impossible de présenter séparément" (p. xi). Une remarque similaire apparaît dans le rapport du recensement de 1881 . On notera que ces brèves mentions de l'expression division du travail n'en font pas un principe classificatoire et n'apparaissent qu'à propos des établissements industriels, jamais à propos des occupations proprement dites.

28 On trouvera un exposé beaucoup plus détaillé des transformations qu'ont connues les classifications occupationnelles utilisées de 1666 à 1901 dans Jean-Pierre Beaud et Jean-Guy Prévost, "Classement statistique, représentations sociales et discours économique: les métiers et professions dans les recensements canadiens", Interventions économiques, no 24,1992 , p. 129-149. 
approximatif, ces appellations constituaient une liste on ne peut plus pittoresque (on y retrouve des manchonniers, des bimblotiers, des fabricants de mélodions, de peignes, d'instruments de mathématiques, de salaratus, ...), mais parfaitement dépourvue d'utilité, tout à fait inapte à la comparaison, à l'analyse ou à l'inférence. Qu'aucune réflexion critique n'ait présidé à la constitution de ces nomenclatures est démontré par le fait que le mode de présentation des occupations utilisé en 1851 et 1861 était identique à celui mis en oeuvre par Jean Talon en 1666. En 1871 et 1881 , les métiers et professions seront regroupés en quelques grandes classes (agricole, commerciale, domestique, industrielle et professionnelle): ce découpage, mis au point par William Farr lors du recensement britannique de 1861, a certes déjà été décrit comme une "classification par fonctions économiques, en termes de larges secteurs"29. Mais il faut ajouter que ces classes ne répondent pas à des définitions strictes et ne connaissent pas de frontières nettes. Elles sont plutôt construites à partir d'un noyau auquel s'agglutinent des occupations qui, d'une façon ou d'une autre, s'en approchent: ainsi, les vétérinaires sont intégrés à la classe agricole, les horlogers, bijoutiers et épiciers à la classe industrielle, même si plusieurs d'entre eux, se livrant à l'échange plus qu'à la transformation, ne répondent nullement à la définition que l'on donne par ailleurs de l'activité industrielle. On ne peut pas dire qu'avant 1891 l'occupation constitue véritablement une variable économique: on s'enquiert alors du métier d'une personne comme de son état civil, de son origine ou de sa religion; plus précisément, il n'y a, de la part des responsables du recensement, aucun travail d'abstraction pour faire de ces données autre chose qu'un miroir des représentations qui ordonnent spontanément le champ social. Dans les rapports des recensements de 1851 et 1861 , on va même jusqu'à séparer graphiquement les titres des fonctions de nature publique (juges, évêques, maires, etc.) des appellations relatives aux autres activités, introduisant ainsi dans les tableaux une distinction hiérarchique. Cette césure sociale disparaît en 1871, mais l'indétermination qui entoure alors le classement des journaliers (leur statut commun doit-il conduire à les assimiler ou leurs activités diverses à les distinguer?) témoigne de la persistance de cette conception sociale plutôt qu'économique de l'occupation ${ }^{30}$.

C'est seulement à partir de 1891 qu'une réflexion critique remettra en cause ces manières de classer les occupations et que, pour la première fois, une conceptualisation, une perspective théorique présideront au découpage des métiers et professions. Le manuel d'instructions critiquera sévèrement la pratique passée,

29 R. S. Szreter, "The Registrar-General's Social Classification of Occupations", The British Journal of Sociology, 35, 1984, p. 542.

30 Classer les journaliers suivant leur activité reviendrait à les inclure dans la classe à laquelle appartiennent leurs employeurs; c'est d'ailleurs ce que semble recommander implicitement le manuel d'instructions de 1881. En pratique, les journaliers se retrouvent dans les sommaires parmi les occupations non classées, ce qui permet de croire que leur statut (les journaliers constituent l'échelon le plus bas de la hiérarchie occupationnelle) l'a emporté, dans l'esprit des recenseurs, sur la nature particulière de leur activité. 
reprochant aux tableaux des recensements antérieurs de comporter "nombre de termes indéfinis qui, en l'absence de renseignements plus précis, rendent impossible pour le département une classification qui aurait eu toute l'utilité voulue" 31 . Désormais, le concept de division du travail, emprunté à la science économique, constituera la pierre angulaire de la classification des occupations: l'objectif ne sera plus de refléter l'univers du travail mais d'appréhender le mouvement de la division du travail, d'où les appels pressants à la plus grande précision possible.

Mais le rejet de la conception "réflexive" et la recherche de la précision dans la désignation du travail accompli par les répondants ne sont pas les seuls effets de l'adoption du concept de division du travail; la structure même de la nomenclature, c'est-à-dire le regroupement de l'ensemble des postes élémentaires en quelques grandes classes, se veut, elle, une représentation de la division générale ou globale du travail dans l'ensemble de la société canadienne:

La première (classe) comprend les producteurs de matières premières; la seconde, les agents distributeurs; la troisième, les fabricants; dans la quatrième et la cinquième classes sont comprises toutes les personnes engagées dans le service personnel et domestique, et celles aussi engagées dans le service professionnel; la sixième comprend les occupations non-productives ${ }^{32}$.

Chacune des classes est ici distinguée en fonction de sa position au sein ou vis-à-vis du processus de production et c'est selon sa place dans la chaîne productive (ou par rapport à celle-ci) qu'est classée chaque occupation. On peut parler à ce propos d'une représentation systémique de l'activité économique. Le point de vue qui préside au classement des occupations est celui de la science économique et l'intérêt qu'offrent les statistiques de l'occupation tient à ce qu'elles permettent de comparer le mouvement général de la division du travail au Canada à celui qu'on retrouve en d'autres pays. Johnson note par exemple que la classe agricole demeure beaucoup plus importante au Canada que ce n'est le cas en Grande-Bretagne et qu'à l'inverse la classe industrielle y est, toutes proportions gardées, nettement moins lourde que dans les pays d'Europe ${ }^{33}$. Archibald Blue, qui sera en charge du recensement de 1901, poursuivra dans la même veine: "Le point important des statistiques concernant les espèces d'occupation, c'est la preuve qu'elles fournissent du développement qu'a pris la division du travail, particulièrement dans la classe manufacturière et dans celle du transport ${ }^{34}$." Le rôle central que joue l'opposition entre travail productif et travail non productif dans

31 Ministère de l'Agriculture, Manuel contenant l'"Acte du recensement" et les instructions aux officiers employés à faire le troisième recensement du Canada , p. 14.

32 Ministère de l'Agriculture, Recensement du Canada 1890-91, vol. II, 1893, p. vii.

33 Ministère de l'Agriculture, Bulletin no 18, 1893, p. 37-38.

34 Ministère de l'Agriculture, Recensement et statistiques. Bulletin no 1, Employés à gages par occupations, 1907, p. x. 
l'esprit des statisticiens canadiens apparaît clairement dans ce commentaire de Blue sur la structure du nouveau découpage:

Quatre des groupes énumérés dans ce tableau sont, à vrai dire, des nonproducteurs. Ils travaillent, mais leur travail ne produit pas d'article qui puisse être mis en vente sur le marché. Ce sont les soins personnels et domestiques, les services divers, les professions et les catégories du commerce et du transport. Les autres cinq sont l'agriculture, les pêcheries et la pêche, les forêts et les bois, les manufactures et les arts mécaniques et les usines. Ce sont des catégories productrices; leurs travaux sont visibles et le résultat appréciable dans le commerce mondial. Exprimées en statistiques, ces catégories représentent le capital, les recettes, les produits qui dépendent de l'un ou de l'autre, et qui représentent la partie la plus tangible des affaires du pays ${ }^{35}$.

En matière d'économie politique, les statisticiens canadiens font même preuve d'une orthodoxie rigoureuse. Ainsi, alors que William Farr, bien qu'il prétendît reprendre la distinction classique, définissait le travail productif par un vague critère d'utilité sociale (l'inclusion des membres du clergé dans le groupe des producteurs était justifiée par l'utilité de leur "production spirituelle"), Blue demeurait fidèle au point de vue smithien, suivant lequel le travail productif est seulement celui "which adds to the value of the subject upon which it is bestowed"36.

\section{Des petites et faibles choses naîtront les grandes...}

On observe des transformations similaires dans la statistique des établissements industriels. Les recensements de 1871 et 1881 contiennent des données sur le nombre d'établissements industriels, "le capital engagé, le nombre de bras employés, le montant des salaires, la valeur des matières premières et les valeurs totales des produits" ${ }^{\prime 3}$. Le classement des établissements est alphabétique et s'apparente, par son aspect anecdotique, à celui des occupations: ainsi, les tableaux nous révèlent par exemple qu'à travers le pays 11 personnes travaillaient dans des moulins à broyer les os, que le capital investi dans des fabriques de tables de billard s'élevait à $8000 \$$ et que la valeur totale des produits des fabriques de rouets s'établissait à $238812 \$$, mais le tout renvoie l'image d'une société

\footnotetext{
35 Ministère de l'Agriculture, Recensement et statistiques. Bulletin no 11, Population du Canada par industries et professions, 1910, p. 23-24.

36 A. Smith, An Inquiry into the Nature and Causes of the Wealth of Nations, Chicago, The University of Chicago Press, 1976, p. 351. C'est dans l'appendice du rapport général du recensement de 1861 que Farr écrit notamment: "The highest Officers of Government are correctly classed among producers" (Census of England and Wales for the Year, 1861, p. 229). Dans Wealth of Nations, Smith écrit pour sa part: "The sovereign, for example, with all the officers both of justice and war who serve under him, the whole army and navy, are unproductive labourers" (p. 352).

37 Ministère de l'Agriculture, Recensement de 1871, vol. III, 1873, p. xi-xiii.
} 
préindustrielle où domine encore l'artisanat. C'est en 1891 que, pour la première fois, l'on se renseignera sur le nombre de machines à vapeur utilisées dans l'industrie; parmi les distinctions introduites, notons celles entre capital permanent et capital en circulation et entre capital fixe et capital actif ${ }^{38}$. Mais le recensement des industries de 1891 sera, à l'instar des autres statistiques produites sous la gouverne de George Johnson, sévèrement contesté: aussi est-il difficile de savoir si "l'augmentation considérable dans le nombre des établissements industriels durant la dernière décade (sic)" est, comme le prétend le statisticien du Dominion, due au "mouvement tendant à spécialiser ou séparer les industries ${ }^{39 "}$ ou si elle s'explique par la volonté de ce dernier de "grossir (...) la liste des triomphes de la politique nationale $40 "$. Il n'y avait pas, en effet, de norme fixée quant au nombre de personnes qui devaient être réunies pour que l'on puisse parler d'établissement industriel et de nombreux témoignages ont été cités à la Chambre des communes qui tendent à mettre en doute l'impartialité de Johnson à cet égard ${ }^{41}$. Ce n'est qu'à l'occasion du recensement suivant, en 1901, que l'on trouve trace d'une conceptualisation du terme d'industrie. Le commissaire du recensement, Archibald Blue, écrit notamment:

C'est une anomalie de considérer comme un établissement industriel toute chambre ou boutique dans laquelle une ou deux personnes sont employées, et on ne peut accepter cette définition quand tout, dans l'industrie, tend vers la concentration du capital, de l'administration, de l'habileté et du travail. Il y a sans doute des raisons pour connaître la variété et la valeur du travail individuel; mais dans tout plan bien conçu ayant pour objet d'obtenir la statistique des manufactures, l'idée de fabrique doit représenter une entreprise moderne qui progresse. L'idée de manufacture doit avoir ses limites comme l'idée de ferme a les siennes; ainsi nul ne songerait à considérer comme une ferme une pièce de jardin ${ }^{42}$.

38 Ministère de l'Agriculture, Bulletin no 8, Industries manufacturières, 1892 et Bulletin no 10, Industries manufacturières, 1892.

39 Ministère de l'agriculture, Recensement de 1890-91, vol. III, 1894, p. vii.

40 Débats de la Chambre des communes, 1893, p. 1142. Le député libéral Casey soutient que les énumérateurs étant payés suivant le nombre d'industries qu'ils recensaient, "il était de leur intérêt de découvrir autant d'industries que possible" (ibid.).

41 Les débats de la Chambre des communes de 1893 et 1894 témoignent des attaques qu'a dû subir le recensement des industries de 1891. Jouant sur les deux sens du mot "hand", utilisé dans le recensement pour désigner le nombre de personnes travaillant dans une industrie, le député Lavergne relate le cas de Madame Marie Terroux, du village d'Arthabaskaville, "who is fortunate enough to be at the head of two industries, a knitting establishment and a dressmaking establishment. She is so entered in the census and she has two hands for two indusries; presumably her left hand and her right" (Débats de la Chambre des communes, 1894, p. 4128.

42 Ministère de l'Agriculture, Quatrième recensement du Canada 1901, vol. I, 1902, p. ix. 
Les instructions aux énumérateurs stipulent d'ailleurs que "nul établissement manufacturier ou fabrique ne sera ainsi reconnu comme tel pour les fins du recensement s'il n'emploie au moins cinq personnes, soit dans l'établissement même, soit comme ouvriers à la pièce employés en dehors de l'établissement ${ }^{43}$. Le classement alphabétique qui avait été utilisé lors des recensements précédents est délaissé au profit d'un index approuvé par l'Association des manufacturiers canadiens ${ }^{44}$ et d'un classement des établissements en 15 grands groupes prenant pour critère tantôt le produit, tantôt la matière première ${ }^{45}$.

C'est la prise de conscience du phénomène de la concentration qui semble avoir été le ressort de cette remise en cause de l'enregistrement statistique de l'industrie. À cet égard, il est intéressant de noter que, dès 1894, Johnson, ayant sans doute à l'esprit l'avance industrielle de l'Angleterre et des États-Unis, écrivait, à propos de la multiplication des petites industries:

Elles ont pris racine, et l'avenir dira si elles ont suivi ici la règle suivie par elles dans d'autres pays, en se développant en établissements plus considérables. (...) plus tard, quand le développement en de grandes industries se sera accompli, le peuple canadien (pourra) jeter un regard en arrière sur la période des "petites et faibles choses", et montrer de quels humbles commencements la vie industrielle du pays est sortie ${ }^{46}$.

Le rapport du recensement de 1911 témoigne de ce que ce développement était en train de s'accomplir: ainsi, Blue écrit que la diminution du nombre des industries employant moins de cinq personnes s'explique en partie par le fait "que les industries moindres ont été réunies aux plus grandes industries sous une même direction ${ }^{47 "}$. Déjà, en 1901, il n'avait pas cherché "à obtenir de données sur la production de beurre et de fromage fabriqués à la maison, vu que l'établissement

43 Ministère de l'Agriculture, Manuel contenant "l'Acte concernant le recensement" et les instructions aux officiers du quatrième recensement du Canada, 1901, p. 27-28.

44 Le recensement des manufactures de 1901 fut approuvé et endossé par l'Association des manufacturiers qui invita tous ses membres à répondre consciencieusement en les assurant du caractère confidentiel des renseignements recueillis. Voir à ce propos, Ministère de l'Agriculture, Quatrième recensement du Canada 1901, vol. III, 1906, p. vii.

45 Les quinze groupes en question étaient: (1) produits alimentaires, (2) industries textiles, (3) produits du fer et de l'acier, (4) bois et ses produits manufacturés, (5) cuir et ses produits finis, (6) papeterie et imprimerie, (7) liqueurs et breuvages, (8) produits chimiques et composés, (9) produits de l'argile, du verre et de la pierre, (10) métaux et produits des métaux autres que l'acier, (11) tabac et manufactures de tabac, (12) voitures pour transport sur terre, (13) vaisseaux pour transport par eau, (14) industries diverses et (15) industries manuelles.

46 Ministère de l'Agriculture, Recensement de 1890-91, vol. III, 1894, p. vii..

47 Ministère de l'Industrie et du Commerce, Cinquième recensement du Canada 1911, vol. III, 1913, p. vii. 
des beurreries et fromageries a presque complètement détruit ces industries domestiques $^{48 " .}$

\section{Économie politique et statistique: la rencontre}

C'est à l'occasion des recensements de 1891 et 1901 que l'on retrouve également les premières références explicites ou non à des théories économiques. Ainsi, et c'est la première fois que l'on relève un raisonnement de ce genre dans un document produit par un statisticien canadien, Johnson établit une corrélation entre l'augmentation de la productivité et celle des gages, allant même jusqu'à affirmer que "l'application de la science de l'invention et de la main-d'œuvre habile aux arts de production a eu pour effet que le produit a augmenté, que la part du capital revenant au propriétaire a relativement diminué, mais que la part de l'ouvrier habile a augmenté absolument et proportionnellement à ce produit ${ }^{49 "}$. Certes, le propos est optimiste (Johnson écrit en 1892 , en pleine "grande dépression") ${ }^{50}$, il conforte le gouvernement conservateur et ne s'appuie en rien sur une démonstration rigoureuse. Johnson se distingue toutefois très nettement de ses prédécesseurs en ce qu'il ne se contente pas de mesurer la prospérité au moyen d'un indicateur privilégié, ni même de plusieurs; il cherche plutôt, reprenant une théorie dont il ne cite pas l'auteur, à lier entre elles plusieurs variables, établissant ainsi des inférences qui ne sont pas simplement la projection de tendances déjà observées (ce que faisait Hutton en prédisant au Canada des succès démographiques supérieurs à ceux de ses voisins), mais constituent un modèle explicatif. Du reste, cet optimisme est tempéré: une autre "première" du recensement de 1891 tient à ce qu'il cherchait à offrir une mesure (bien imparfaite) du chômage, puisqu'on y demandait à tous ceux qui avaient une occupation s'ils avaient travaillé au cours de la semaine précédant le recensement ${ }^{51}$. Dans un rapport paru en 1907, Ernest $\mathrm{H}$. Godfrey $^{52}$ revient sur le même thème, écrivant :

48 Ministère de l'Agriculture, Quatrième recensement du Canada 1901, vol. I, 1902, p. ix.

49 Ministère de l'Agriculture, Bulletin no 10, Industries manufacturières, p. 19.

50 Les historiens de l'économie canadienne décrivent habituellement les années 18731896 comme la Grande Dépression (laquelle fut suivie par le "take-off" des années 18961914). Voir par exemple Norrie K. et D. Owram, A History of the Canadian Economy, Toronto, Harcourt Brace Jovanovich, 1991, p. 293-298. Certains auteurs ont mis en doute cette périodisation tranchée: voir notamment G.W. Bertram, "Economic Growth in Canadian Industry: 1870-1915", dans W.T. Easterbrook and M.H. Watkins (dir.), Approaches to Canadian Economic History, Toronto et Montreal, McClelland and Stewart , 1967, p. 74-98.

51 Il est à noter que ces renseignements n'ont pas été publiés à l'époque. Quelques historiens ont toutefois tenté d'utiliser ces données pour tracer un portrait du chômage à cette époque. Voir par exemple P. Baskerville et E. Sager, "The First National Unemployment Survey: Unemployment and the Canadian Census of 1891", Labour/Le Travail , 23, 1989, p. 171-178. Il semble qu'en cette matière Johnson ait été quelque peu visionnaire, puisqu'il faudra attendre 1921 pour que les statisticiens se préoccupent à 
Les économistes prétendent avoir établi l'existence d'une loi par laquelle, si la part absolue de profits revenant au capital peut augmenter en proportion de l'augmentation de ce capital, la part relative diminue, tandis que la part du travailleur augmente à la fois relativement et de façon absolue ${ }^{53}$.

Il attribue la formulation de cette loi à l'économiste américain Edward Atkinson ${ }^{54}$ et soutient que l'examen des statistiques récentes tend à démontrer sa validité. Chez Blue également, l'on trouve des références à des auteurs économiques alors en vogue: ainsi, il invoque l'ouvrage du journaliste John Beattie Crosier, The Wheel of Wealth (lequel vulgarise les thèses de Smith) à l'appui de sa conception du rôle de la division du travail dans l'accroissement des richesses et oppose à l'interprétation faite par le britannique Hobson du travail des femmes des arguments que l'on qualifierait aujourd'hui de "progressistes ${ }^{55 " .}$

nouveau de la mesure du chômage. Voir à ce sujet $\mathrm{O}$. Sautter, "Measuring Unemployment in Canada: Federal Efforts before World War II", Histoire sociale/Social History, 15, 1982, p.475-489, et K. Farrall, "Theories, Concepts and the Art of Measurement: Economic Theories' Relationship to the Labour Force Survey Concepts", Notes on Labour Statistics , 1973, Statistics Canada.

52 E. H. Godfrey, fellow de la Royal Statistical Society depuis 1901, entre au service du Census and Statistics Office en 1907. En 1912, il sera membre de la commission départementale mise sur pied pour réformer l'appareil statistique. Il sera en 1913 le premier délégué canadien à l'Institut international de statistique.

53 Census and Statistics, Bulletin II, Manufactures of Canada, 1907. Godfrey cite le texte d'une conférence d'Atkinson intitulée "What Makes the Rate of Wages?" et prononcée à Montréal en 1882 dans le cadre d'un colloque sur l'économie organisé par la British Association for the Advancement of Science. Ce texte, extrait d'un livre intitule The Distribution of Products, or the Mechanism and the Metaphysics of Exchange, fut publié dans l'un des premiers ouvrages canadiens trailtant d'économie politique, Canadian Economics, Montreal, Dawson Brothers, 1884, p. 277-289. Étant donné la proximité des termes utilisés, il est permis de croire que c'est de ce texte que s'inspirait Johnson en 1892.

54 L'économiste américain Edward Atkinson (1827-1905), spécialiste du problème de la détermination des salaires, jouissait d'une certaine notoriété à l'époque, puisqu'il fit l'objet d'une biographie dans l'édition de 1925 du Palgrave Dictionary of Economics.

55 Dans Evolution of Modern Capitalism (1894), Hobson soutenait que "les exigences de la vie de fabrique sont incompatibles avec la position d'une bonne mère, d'une bonne femme ou de toute personne capable de créer une famille" (p. 320, cité par Blue, Employés à gages par occupations, p. xxv ). Blue commente: "On se plaît à dire que la vocation naturelle de la femme est celle de femme de ménage ou de mère de famille, et que par conséquent elle ne devrait pas être encouragée à entrer en concurrence avec l'homme dans le domaine des autres occupations et ainsi réduire par son travail inférieur et à meilleur marché les chances de celui-ci de se procurer de l'emploi à des gages qui lui permettent de vivre. (...) Mais est-elle moins capable que la plupart des hommes de s'acquitter d'un travail qui ne demande aucune force physique (...)? Est-elle en quelque manière inférieure à l'homme dans un emploi où une main instruite et un esprit renseigné assurent l'expédition et l'exactitude dans le travail à accomplir? Elle est prompte à saisir, elle est pratique et elle possède à un très haut degré le tempérament fort et nerveux 
S'il faut voir au cours des années 1891-1911 une imprégnation progressive du discours statistique par les préoccupations, les objets et les concepts propres à l'économie politique, c'est de la même période qu'il faut dater l'apparition de cette discipline sur la scène intellectuelle canadienne. Non qu'il n'y ait eu de débats économiques proprement canadiens au cours du XIXe siècle: au contraire, tout l'argumentaire libre-échangiste développé en Grande-Bretagne et en France par les Smith, Say, Bastiat, Cobden et Bright était fort connu et repris avec conviction par des groupes comme l'Association pour le libre-échange ou encore par des politiciens comme le leader libéral Cartwright ${ }^{56}$; et l'on pourrait ajouter que des auteurs aussi éminents que John Rae et Goldwin Smith ont longuement séjourné au Canada. Mais comme le démontre Craufurd Goodwin, l'économie politique n'y avait en aucune façon le statut d'une discipline scientifique: en effet, il n'existait alors au Canada ni chaires d'économie dans les universités, ni société savante en réunissant les praticiens, et un auteur comme Rae, peut-être l'économiste le plus célèbre à avoir vécu au Canada au XIXe siècle, devait se résoudre à publier à l'extérieur du pays ${ }^{57}$. Aucun des participants canadiens au colloque montréalais de 1884 portant sur l'économie canadienne ne revendiquait le titre d'économiste ou de statisticien; en 1886, la Commission d'enquête sur les relations entre le capital et le travail ne comptait en son sein aucun économiste et certains témoins, parmi lesquels Archibald Blue, alors assistant commissaire de l'Agriculture et secrétaire du Bureau ontarien des industries, et Frederick Nichols, secrétaire de l'Association canadienne des manufacturiers, plaideront pour un développement de la collecte de données statistiques, seul fondement possible, selon eux, d'une investigation économique sérieuse ${ }^{58}$. Ce n'est qu'en 1889 que l'Université de Toronto mettra sur pied une chaire "d'économie politique et d'histoire constitutionnelle"; le tour de Queen's viendra deux ans plus tard et il faudra attendre 1901 pour que McGill en crée une ${ }^{59}$. Selon un historien de la discipline, le véritable acte de naissance de cette dernière est la mise sur pied, en 1913, de la première association professionnelle d'économistes, la Canadian Political Science Association, à quoi il faut ajouter la parution (entre 1913 et 1917) de l'encyclopédique Canada and its Provinces, dont les chapitres économiques constituent "les plus importantes

qui ne se fatigue que le travail fini ou l'épreuve passée. On peut donc dire que la femme possède des qualités spéciales qui la recommandent à la considération dans un système tendant au rajustement des gages" (ibid., p. xxvi).

56 Voir C. D. W. Goodwin, Canadian Economic Thought. The Political Economy of a Developing Nation 1814-1914, Durham (N.C.), Duke University Press, 1961, p. 5968.

57 Rae, qui vécut au Canada de 1822 à 1848, y fut employé comme maître d'école. Il y écrivit l'un de ses principaux ouvrages (Statement of Some New Principles on the Subject of Political Economy...), lequel parut à Boston en 1834. Voir Goodwin, Canadian Economic Thought, p. 122-127.

58 Commission royale d'enquête sur les relations entre le capital et le travail (1888). Pour les points de vue de Nichols et de Blue, voir le volume sur la province d'Ontario, p. 213 et 100 .

59 Goodwin, Canadian Economic Thought, p. 152-175. 
contributions à la littérature économique canadienne avant $1920^{60 "}$. On retrouve parmi les membres fondateurs de la C.P.S.A. Adam Shortt, "the real founder of Canadian economics ${ }^{61}$ ", et Robert $\mathrm{H}$. Coats, considéré lui aussi par les historiens de la discipline comme l'un de ses "founding fathers ${ }^{62 "}$. Tous deux ont siégé à la commission départementale mise sur pied pour réformer le système statistique et contribué à Canada and its Provinces; le premier, après avoir été titulaire de la chaire d'économie à Queen's, est président de la Commission de la fonction publique depuis 1908, le second est rédacteur de la Labour Gazette, sous les ordres de W.L. Mackenzie King, lui-même titulaire d'un doctorat en économie de l'Université de Chicago. Mais nous franchissons ici la borne que nous nous étions fixée: cette association entre Shortt et Coats montre bien qu'après 1912-1913 science économique et collecte de données statistiques vont de pair ${ }^{63}$. En même temps s'est effectuée une distinction très nette entre statistiques démographiques et statistiques économiques: celle-ci est on ne peut mieux illustrée par Coats luimême lorsque, dans l'un de ses nombreux avant-projets pour la mise sur pied d'un système statistique national, il renverse l'ordre traditionnel, qui accordait la priorité aux statistiques de la population, pour affirmer catégoriquement que les statistiques les plus importantes d'un pays sont celles ayant trait à son économie.

\author{
Jean-Pierre BEAUD et \\ Jean-Guy PRÉVOST \\ Département de science politique \\ Université du Québec à Montréal
}

\title{
Résumé
}

Jusqu'à la fin du XIXe siècle, l'activité principale des statisticiens était le recensement des personnes et leur attention se portait essentiellement sur les statistiques de la population. Plus précisément, la croissance démographique était tenue pour l'indicateur privilégié de la prospérité du pays. Le début du XXe siècle correspondra toutefois, pour la plupart des appareils statistiques nationaux, à une transformation profonde: désormais, les données relatives à la production et aux échanges occuperont la première place et les spécialistes de celles-ci, les économistes, prendront les postes de commande. Cet article expose comment cette mutation s'est opérée dans un cas précis, celui du Canada; il s'agira en somme de

60 K.W. Taylor, "Economic Scholarship in Canada", The Canadian Journal of Economics and Political Science, 26, 1960, p. 9.

61 Ibid., p. 8. Voir aussi, pour des jugements similaires, Goodwin, Canadian Economic Thought, p. 162 et H. Innis, "The State of Economic Science in Canada", Commerce Journal, 1933, p. 6.

62 Taylor, "Economic Scholarship in Canada", p. 12.

63 Coats dira quelques années plus tard: "The final function of the Dominion Bureau of Statistics is that of laboratory for social and economic research" ("Fifty Years of Statistical Progress", p. 83. 
repérer les transformations que connaîtront certains objets d'enquête, l'apparition de nouveaux champs d'investigation et surtout l'émergence rapide, dans le discours produit par l'appareil statistique, de concepts clés de l'économie politique classique.

Mots-clés: statistique, population, démographie, prospérité, appareil statistique national, production, économie politique.

\section{Summary}

Until the end of the 19th century, government statisticians were mainly concerned by censuses of population; demographic statistics attracted almost all of their interest. One could even say that demographic growth was considered then as the foremost indicator of a country's prosperity. In the beginning of the 20th century, however, most national statistical agencies experienced profound change: from then on, data relating to production and exchange would hold first rank; economists, being acknowledged specialists on these matters, would rapidly rise to the head of these agencies. This paper seeks to expose how such a transformation occurred in a specific case, that of Canada: it will do so by tracing the changing approaches to selected fields of inquiry, the emergence of new fields for data collection, and, above all, the rapid emergence, of some of the key concepts of classical political economy in duscussions among statisticians.

Key-words: Statistics, population, demography, prosperity, national statistical agency, production, political economy.

\section{Resumen}

Hasta el final del siglo XIX, la principal actividad de los estadísticos era el censo de las personas y su atención iba dirigida esencialmente a las estadísticas de población. Tanto es así que el crecimiento demográfico era considerado el indicador privilegiado de la prosperidad del país. El comienzo del siglo XX marcará empero, para la mayoría de los aparatos estadísticos nacionales, una transformación profunda. Desde entonces los datos relativos a la producción y al intercambio habrían de ocupar el primer lugar, y sus especialistas, los economistas, pasarían a ocupar las posiciones de primer orden. Este artículo expone cómo se produjo esta mutación en un caso preciso, el Canadá. Se tratará en suma de captar las transformaciones sufridas por ciertos objetos de investigación, y sobre todo, el rápido surgimiento en el discurso producido por el aparato estadístico, de conceptos claves de la economía política clásica.

Palabras claves: estadística, población, demografía, prosperidad, aparato estadístico nacional, producción, economía política. 